\section{Some Points}

IN

\section{THE SURGICAL TREATMENT OF DISEASES OF THE GALL-BLADDER AND BILE DUCTS * \\ BY}

\author{
R. P. ROWLANDS, M.S., F.R.C.S. \\ SURGEON TO GUY'S HOSPITAL
}

I believe that nearly all the diseases of the biliary tract are directly or indirectly due to blood-borne infections, causing cholecystitis with the consequent deposition of gall-stones in the gall-bladder, where the bile becomes so greatly concentrated. It is acknowledged that the gallbladder is the only considerable factory for the formation of gall-stones, which rarely form elsewhere. We have reason to believe that mankind has suffered from cholelithiasis from time immemorial, as is well illustrated by the recent discovery of gall-stones in Egyptian mummies, which can-be seen at the Royal College of Surgeons. It is sad to think of the terrible pain and suffering which gall-stones have caused men and women throughout the ages. Medicine had little to offer beyond imperfect relief of pain by sedatives. As late as 1890 a distinguished physician seriously advocated massage as a means of expelling stones through the bile ducts into the duodenum. Nature occasionally succeeded in getting rid of stones, but with a high mortality and long suffering, for Nature is a poor substitute for modern surgery.

\section{Early Operations on Gall-Bladder}

Although Petit suggested cholecystotomy in 1733, the operation was not actually performed until 1867. In that memorable year Lister published his epoch-making discoveries which gave birth to modern surgery, and made him the greatest benefactor of the human race. Before his time surgery could not safely interfere beyond opening abscesses or slitting sinuses for the extraction of stray stones. A little more than fifty years ago a few surgeons adopted Lister's methods, and ventured to embark upon pioneer work in the upper abdomen, but for a long time few dared to trespass beyond the gall-bladder, from which they were content to remove stones with or without drainage-cholecystostomy or cholecystotomy. In 1882 Langenbuch first successfully removed the gall-bladder, but by 1890 only forty-seven cholecystectomies had been recorded.

The surgery of the bile ducts was naturally of slower development, although choledochotomy was first successfully performed in 1889 by Knowsley Thornton. Since then, and especially during the present century, asepsis and technical improvements have made surgery so comparatively safe that operations upon the biliary tract have multiplied with great rapidity. In fact, there is now considerable danger that too many operations may be performed and too many gall-bladders drained or removed, sometimes by ill-trained operators. Let us not repeat the errors of the past and bring the surgery of the gallbladder into the disrepute which justly attached itself at one time to ovariotomy, appendicectomy, and colectomy.

We must never forget that the present great success of operations upon the gall-bladder and bile ducts, carried out by experts, can only be obtained by those who take the greatest pains to train themselves in this difficult branch of surgery, and to observe most scrupulously the necessary precautions. Much can be learnt from a careful study of the anatomy, physiology, and pathology of the

* An address delivered to the Cardiff Medical Society. bile apparatus, and especially the important and confusing changes in the surrounding tissues produced by inflammation. But the perfect skill and judgement which can save a surgeon from the pitfalls that beset his path can only be attained by watching, assisting, and operating under the supervision and encouragement of a master in this special branch of surgery. Thus with perfect training and greater care we ought to see and hear less of those injuries to the bile ducts which demand difficult reconstructive operations. Here prevention is far better than cure.

\section{Diagnostic Methods}

Many advances have been made in recent years in the diagnosis of cholecystitis and gall-stones. If it be true that about 10 per cent. of the population have gall-stones, and many more have cholecystitis, there are plenty of opportunities of testing both old and new methods. As usual, there is some danger of forgetting the old and relying too much on the new. A careful and repeated study of the history, symptoms, and clinical signs is still valuable and indispensable. These are so characteristic that they generally lead us to correct conclusions, which may, however, need confirmation by cholecystography. Simple radiography shows the shadows of some opaque gall-stones and of thick-walled or calcified gall-bladders. With the aid of the opaque meal it may indicate that a distended or retracted gall-bladder is pressing or dragging upon the duodenum, stomach, or colon.

Cholecystography is a great advance, and often reveals cholecystitis or small non-opaque gall-stones. It is particularly useful in showing obstruction of the cystic duct. It is invaluable in the differential diagnosis of diseases of the gall-bladder from those of the alimentary and urinary tracts. Above all, it of ten gives the patient that confidence in the diagnosis which he desires before consenting to operation. The discovery of pathological changes in the bile obtained through the duodenal tube sometimes helps materially in the diagnosis of difficult cases.

\section{Differential Diagnosis}

In passing, I may mention here some of the difficulties that may be met with in the differential diagnosis.

Severe acute inflammation of the gall-bladder, perhaps with perforation, may be mistaken for perforated duodenal or gastric ulcer, or acute pancreatitis. The gall-bladder is not always palpable and recognizable as such in these cases; it may be high up or far back in the right loin. Sometimes it is so low as to be mistaken for appendicitis with abscess. When there is grave doubt it is safer to explore without delay. Large renal swellings, especially hydro- or pyo-nephrosis, may be dull in front and thus mistaken for a distended gall-bladder. Hydatid cyst projecting from the under surface of the liver, from the kidney, or from the pancreas, may also be mistaken for a distended gall-bladder. In one case I mistook an enlarged gummatous pedunculated accessory lobe of the liver for the gall-bladder. An enlarged adherent gall-bladder has been mistaken for inoperable cancer or ulcer of the stomach, and vice versa.

In most of these cases the symptoms, and particularly the radiographic findings, are invaluable. Cholecystitis, with or without small gall-stones, is constantly confused with duodenal ulcer; and, even now, with all the help we can get from clinical, radiographic, and chemical examinations we sometimes have to explore the abdomen before we can complete the diagnosis and cure the patient.

I have a middle-aged man now at Guy's who had been confidently treated for duodenal ulcer for many years. There was a definite history of severe haematemesis and melaena, and the radiographic and chemical examinations confirmed the diagnosis. At the operation there was no 
sign of any ulcer anywhere, but an enlarged, slightly thickened, kinked gall-bladder was found and removed. Very small black stones were then found in the gallbladder and cystic duct. Another difficult case may be mentioned in which cholecystitis with gall-stones was mistaken for duodenal ulcer.

\section{Illustrative Case}

A man, aged 50 years, first seen by me in May, 1926, gave a history of a severe attack of enteric in 1903 . For the last twelve years he had had attacks of indigestion of the duodenal type, and was seen by several distinguished physicians, who all suspected a duodenal ulcer. $\mathrm{He}$ had been $x$-rayed several times, but neither the radiogram nor the screen examinations ever showed an ulcer. Two years ago he saw another physician, who found inarked hyperchlorhydria. An $x$-ray examination then showed some defective filling of the duodenal cap. Cholecystography was not tried. The patient was sent to bed on a duodenal ulcer diet, and he did well, putting on half a stone in weight.

At 9 p.m. on May 20th, 1926, he was seized with very severe pain in the right hypochondrium. In spite of morphine this became much worse about 5 o'clock the next morning. Perforation of the duodenum with local extravasation was feared. I saw him at $\mathbf{8 . 3 0}$ a.m. the same day, and could then just feel the fundus of an enlarged gall-bladder. The diagnosis of acute cholecystitis was then clear, whether a duodenal ulcer was present or not. After the attack had subsided the gall-bladder was removed. There was no evidence of a duodenal ulcer. The gall-bladder was thick-walled, inflamed, and full of stones, one of them impacted in the cystic duct. There was no evidence of any stone in, or obstruction of, the common bile duct. The appendix, which was adherent to the left side of the brim of the pelvis from old inflammation, was removed. Complete recovery followed.

Comment.-This case is interesting, because the patient, a very observant doctor, thought for many years that he had a duodenal ulcer, and the many physicians who saw him agreed ; and yet the history was, to my mind, more suggestive of intermittent attacks of obstruction of the cystic duct, with the dyspepsia that so often accompanies cholecystitis. The pain was severe, came on suddenly and capriciously at any time (especially during exercise), and ceased just as suddenly. Sometimes it interrupted a game of golf, but after a short rest the patient was able to finish the round. Vomiting frequently brought relief. Neither haematemesis nor melaena had ever been observed; occult blood in the stools had never been found. On one occasion only was there defective filling of the duodenal cap, and that was probably due to reflex spasm.

An operation, which was the only means of curing this patient, was deferred for many years owing to an impression that he was suffering from a duodenal ulcer, and because the patient preferred medical to surgical treatment.

\section{Cholelithiasis and Jaundice}

Patients, and even medical men, still have a difficulty in accepting the diagnosis of gall-stones unless there is history of jaundice. But we know that jaundice is a late and rare sign of gall-stones, not to be expected when the disease is limited to the gall-bladder and cystic duct, and often absent even when stones lie in the common bile duct for years. They may not obstruct the bile for a sufficient length of time to cause jaundice.

Biliary colic without jaundice is often mistaken for right renal colic, but the site and distribution of pain and tenderness are very different in the two cases. Biliary colic with jaundice is not always due to stone in the common duct; it may be caused by other things, such as blood clot, daughter cysts, or papillary growth inside the duct. A roundworm may ascend the bile ducts and cause jaundice, and even acute necrosis of the pancreas.
The exact cause of chronic jaundice without history of colic is often difficult to ascertain. The essential thing to decide is whether an operation can or cannot be expected to cure or relieve it. Hence it is important to find out if the gall-bladder is enlarged and can be drained into the stomach, so that jaundice, itching, anorexia, and wasting may be corrected, and life prolonged and made more tolerable. When there is neither history of colic nor palpable enlargement of the gall-bladder, an operation is not hopeful. The jaundice may be due to cirrhosis or carcinoma of the liver obstructing the smaller ducts; or it may be caused by cancer obstructing the common hepatic duct.

It is well known that the gall-bladder is enlarged in all cases of obstruction of the common bile duct except stone-Courvoisier's law. Usually this law is reliable, but occasionally the gall-bladder is enlarged when a stone in the common bile duct is the cause of jaundice, for there may be coincident obstruction of the cystic duct.

\section{INDICATIONS FOR OPERATION}

Obstruction of the Cystic Duct.-This is indicated by attacks of biliary colic, with tenderness and rigidity in the right hypochondrium and often a palpable gall-bladder during or after the attack. Failure to fill the gall-bladder with dye is very strong evidence of obstruction of the cystic duct.

Recurrent or Persistent Irritation and Infection of Gallbladder.-This is indicated by chronic flatulent dyspepsia, pain, and tenderness over the gall-bladder referred to the lower angle of the right scapula. Cholecystographic evidence of defective filling, concentration, and emptying, or of stones in the gall-bladder, is of great and increasing assistance.

Obstruction of Common Bile Duct.-This is evidenced by (1) recurrent attacks of biliary colic with moderate jaundice (these nearly always indicate calculous obstruction, and it is unfortunate that the $x$-ray films very rarely show stones in the common duct), and (2) increasing and persistent chronic jaundice with enlargement of the gallbladder. This latter is generally due to chronic pancreatitis, or carcinoma of the head of the pancreas or common bile duct, but is sometimes caused by stone in the duct. Here great care must be taken to exclude other causes of jaundice, such as cirrhosis or malignant disease of the liver, acute yellow atrophy, and other forms of toxic jaundice, for which an operation is strongly contraindicated.

Stones without Symptoms. - It ought to be evident that the mere demonstration of gall-stones in the gall-bladder is not a sufficient indication for operation. They are very common, and in many they cause no appreciable discomfort, or only such as can be readily relieved by medical treatment ; but when symptoms of cholecystitis or of gallstones recur or persist in spite of medical treatment, it is wise to recommend early operation in order to prevent a high mortality and other serious consequences of delay. It is well to remember that in spite of the extravagant claims of quacks we have no medical means of dissolving or of evacuating gall-stones, although some of these may sometimes pass spontaneously along the ducts, or escape through fistulae into the bowel.

\section{Treatment of the Acute Attack}

As a good general rule it is wise not to operate during an acute attack of colic, jaundice, or fever. It is better and safer to wait for a quiet interval, using the time to investigate and to prepare the patient very carefully. Later, a more radical operation can be safely performed. 
Fortunately the acute symptoms usually subside under complete rest and careful medical treatment, for, unlike the appendix, the gall-bladder and bile ducts rarely set up spreading peritonitis. It is true that occasionally the acute symptoms do not abate, but get worse, and demand emergency operations. Usually these should be limited to drainage of the gall-bladder or common bile duct. Radical operations can, if necessary, be carried out later, when the patient is better, the liver functions are restored, and sepsis has subsided.

It is a great mistake to let many recurrences take place, to allow time for dangerous complications to develop, or the general health to become seriously depreciated. Too long watching and waiting and relying upon drugs and dieting for the relief of symptoms inevitably leads to much suffering and a greatly increased mortality from delayed operation.

\section{Consequences of Delay}

Some of these are: (1) gangrene, suppuration, and perforation of the gall-bladder with local or even diffuse peritonitis, pylephlebitis, septicaemia, and multiple arthritis ; (2) extension of the disease to the common bile duct with jaundice, infective or suppurative cholangitis, with risks of haemorrhage and cholaemia ; (3) extension to the pancreas, causing either acute haemorrhage or chronic pancreatitis, with or without jaundice ; (4) intestinal obstruction by gall-stones ulcerating through into the duodenum or colon; (5) adenoma, papilloma, and cancer from chronic irritation of the gall-bladder and bile ducts ; (6) cardiac degeneration from chronic septic absorption (the damaged heart is often restored by removal of a septic gall-bladder) ; (7) cerebral toxaemia from the same causes may induce mental confusion, depression, and even insanity, which are sometimes overcome by removing the septic source of poisoning.

\section{Pre-operative Treatment}

Much has been done in recent years to make operations safer by improving the preparation of patients, and this applies particularly to the surgery of the bile ducts, which is beset with special dangers.

The patient is kept at rest, preferably in bed, for several days and dieted ; the bowels are regulated, and the colon is washed out at least eight hours before the operation. Plenty of sugar and sodium bicarbonate are given by the mouth ; 5 per cent. glucose in normal saline is given by the rectum, or even intravenously, in all bad cases where failure of the liver functions is feared. Hexamine is given as a biliary antiseptic. All septic sources in the mouth, nose, and throat are treated in time to lessen the risk of pulmonary complications. When the patient is jaundiced and clotting of the blood is delayed, $1 \mathrm{c.cm}$. of a 10 per cent. solution of calcium chloride is injected intravenously for two or three days before the operation. Sometimes it is more convenient to give the calcium chloride intramuscularly -1 grain is dissolved in 10 minims of water and kept in a sterile ampoule ready for injection once a day. If this fails to bring the clotting time to the normal, or if there is marked anaemia, haemoplastic serum or one or more transfusions of blood may be given with great advantage.

\section{The Anaesthetic}

Chloroform is best avoided in these cases, owing to its destructive effects on liver cells. An injection of $1 / 4$ grain of morphine and 1/100 grain of atropine is given fortyfive minutes before the operation. Ether and oxygen are administered. Some prefer gas and oxygen, but this rarely affords enough muscular relaxation.
The Operation

I find that Kocher's incision, which divides the whole width of the rectus muscle and its sheath one inch below the right costal margin, gives by far the best access for cholecystectomy, especially in fat and muscular patients. It makes a difficult operation much easier and safer, because it gives a splendid view for what may prove a difficult and dangerous dissection among the important biood vessels and bile ducts near the portal fissure. It is easy to close, and is rarely followed by ventral hernia, although it allows free drainage at its outer angle. It does not rob the rectus of an adequate nerve supply. For exposing the common duct in late cases of jaundice I choose a right paramedian epigastric incision, which gives the best access to the lower part of the common bile duct, with very little haemorrhage from the parietes.

The abdomen having been opened, the surgeon rapidly but carefully explores, so that he may discover and remove any source of sepsis. It is generally wise to remove the appendix, which is often affected, at the same time. The gall-bladder and bile ducts are examined with special care, so that no stone or disease is overlooked. Stones may rest in the common bile duct for years without causing jaundice. When the common bile duct is dilated, it must be carefully palpated from end to end, and sometimes it is necessary to open it and to pass a large probe through into the duodenum in order to be quite certain that no obstruction is missed.

It is fairly easy to recognize gross disease of the gallbladder, but slight yet important abnormalities can be easily passed over. It is well to look for the following indications of disease ; adhesions between the gall-bladder and the parietes, duodenum, colon, or omentum ; abnormalities of size and shape (intermittent obstruction often causes enlargement, while chronic inflammation generally induces contraction) ; thickening, with change of colour to pink or grey i small stones, often difficult to feel even after the bile has been squeezed out of the gall-bladder (it is especially difficult to feel very small stones in the convoluted cystic duct, which is only one-tenth of an inch in diameter); white spots indicating cholesterosis or strawberry gall-bladder; an excess of fat in the wall of the gall-bladder; enlargement of the sentinel lymphatic gland near the cystic duct (especially important), and often of the glands apon the common bile duct; and, finally, evidence of hepatitis and of peri-hepatitisespecially of subcapsular fibrosis near the gall-bladder.

I am particularly interested in kinks, bands, adhesions, and deformities at the neck of the gall-bladder, which may be concealed by their peritoneal coverings. The junction of the gall-bladder and cystic duct often gets kinked, making a valve similar to the one so often causing hydronephrosis. The pavilion then dilates and becomes a backwater for stasis. A long, pedunculated gall-bladder is apt to twist and to obstruct its neck, resulting in colic, inflammation, or even gangrene. All these cause stasis of bile in the gall-bladder, and may lead to over-concentration, cholecystitis, and the deposition of gall-stones.

Adenoma of the gall-bladder is a fairly common result or cause of chronic inflammation. It is generally seen at the fundus, but $I$ have seen it at other parts-in one case at the neck, resembling a miniature obstructive enlarged prostate. In another, a polypoid adenoma caused intermittent obstruction of the orifice of the cystic duct. Cancer of the gall-bladder is estimated to complicate over 5 per cent. of all cases of gall-stones. The danger of this is greater than that of an operation for gall-stones.

In conclusion, I think that if the clinical evidence clearly incriminates it, it is wise to remove the gall-bladder when it is exposed, for we can easily overlook evidence of small stones, cholesterosis, or intermittent obstruction of the cystic duct. 
Choice of Operation after Exposure of Parts

Disease Limited to Gall-bladder and Cystic Duct.-In this case the choice lies between cholecystectomy and cholecystostomy. It is the ideal of the surgeon to operate while the disease is limited to the gall-bladder and cystic duct, so that he can eradicate it. Fortunately the gallbladder is not indispensable, although it has been shown to have several interesting functions. Certain animals, such as the horse, the deer, and the rat, have no gallbladder, and thousands of human beings who have had it removed get on well without it. Skilful excision in suitable cases carries little risk; therefore we need not hesitate to remove it when this is necessary and the common bile duct is not obstructed. Removal is far better than drainage, for the following reasons. (1) Drainage rarely cures cholecystitis, because the infection lurks deeply in the walls of the gall-bladder. (2) A diseased gall-bladder, being the chief factory of gall-stones is better removed to prevent recurrence. (3) It is extremely difficult to make sure of getting rid of all the stones from the cystic duct and the gall-bladder. It is often easier, cleaner, and therefore safer, to take away the gall-bladder and cystic duct without opening them. (4) Excision of the gallbladder may get rid of an unsuspected early cancer or precancerous condition; the results of such operations are extremely favourable. I have several patients living and well many years afterwards, but it is usually too late to cure a cancer of the gall-bladder which has been recognized before the abdomen has been opened.

The chief indications for cholecystectomy are irreparable wounds and injuries, or diseases limited to the gall-bladder and its duct. The most important of these diseases are: acute, chronic, and recurrent cholecystitis, especially when associated with gall-stones; typhoid infection, making a patient a typhoid carrier; gangrene, perforation, empyema, and hydrops, or mucous fistula of the gall-bladder due to obstruction of the cystic duct by stone, torsion, kink, band, or stricture ; fibroma, adenoma, papilloma, and carcinoma ; and biliary fistula and chronic jaundice due to kinking of the common bile duct following cholecystostomy.

If every gall-bladder is carefully examined after removal I believe that more new growths will be discovered. Many of the adenomata which I have excised contained small black septic stones. I think these adenomata have a very important bearing upon stone and cancer formation.

It is not wise to undertake cholecystectomy when the patient is very ill, old, and feeble, or when the mechanical difficulties and danger of the operation are too great. Cholecystostomy is safer in such circumstances, care being taken to remove all the stones, if possible. If the symptoms recur, secondary cholecystectomy can be performed in more favourable circumstances.

With Spread of Disease to Common Bile Duct.-The extent of operation in these bad cases depends on the general condition and age of the patient, the duration of jaundice, the amount of infection of the bile ducts, the degree of deterioration of the liver functions, and the state of the pancreas. In grave cases all that is urgently required is choledochotomy with drainage of the common bile duct. This will probably rescue the patient from the perils of cholaemia and sepsis, and allow a more radical operation to be done, if necessary, when the patient is better. In less grave cases the stones may also be taken out of the gall-bladder by cholecystostomy. In favourable cases the gall-bladder may be excised; but this should not be attempted when there is chronic jaundice (with consequent risk of uncontrollable haemorrhage) unless the normal coagulation time has been restored by treatment, nor when there is infective or suppurative cholangitis, or hepatic insufficiency. Cholecystectomy should never be performed unless it is certain that the common bile duct is patent.

\section{Operative Results}

The results of operations upon the gall-bladder and bile ducts, performed by good and specially trained surgeons, are very satisfactory, and compare favourably with those obtained in any other branch of surgery.

\section{Mortality}

The death rate is surprisingly low, considering the complicated nature and difficulties of these operations. My results during the last twenty years are as follows. The mortality in 434 cholecystectomies was 1.6 per cent. ; in 116 cholecystostomies 18 per cent. ; and in 116 choledochotomies 12 per cent.

The death rate of cholecystostomy is much higher than that of cholecystectomy, because this operation is generally reserved for bad and late cases. Similarly, the mortality of choledochotomy is naturally high, because the patients requiring it are often bad subjects, who have suffered for many years, who have developed complications such as secondary heart disease, cholangitis, hepatitis, or pancreatitis, and who are peculiarly liable to haemorrhage; above all, they are apt to suffer from hepatic insufficiency, nephritis, and pneumonia. Many of these cases are too late for the best results to be obtained. If they come reasonably early the mortality ought not to be above 5 per cent., but it may be as high as 35 per cent. when infective or suppurative cholangitis has supervened.

The mortality is higher in private than in hospital patients, chiefly because the former wait too long and try a variety of medical conservative cures before they come to operation. Hospital patients cannot afford to wait. For similar reasons, the mortality in men is higher than in women.

\section{Ultimate Results}

Chòlecystostomy affords complete and permanent relief of symptoms in only about 45 per cent. of cases. The persistence or recurrence of symptoms is often due to stones left behind, to the persistence of inflammation in the walls of the gall-bladder, or to the formation of new stones. Secondary cholecystectomy or choledochostomy is often required.

Cholecystectomy affords complete and permanent relief in about 86 per cent. of cases. Müller found the results completely satisfactory in 84 per cent. of stone cases and in 70 per cent. of cases of cholecystitis without stones. Whipple gives similar figures. At least 80 per cent. of my results were good. The more accurate the diagnosis, and the more complete the operation, the better the result. Hurst has pointed out that some temporary recurrence of symptoms may follow cholecystectomy, owing, perhaps, to spasm of the sphincter of Oddi, with dilatation of, and inflammatory changes in, the bile ducts secondary to loss of the gall-bladder. Under these conditions spasmodic pains may develop and be attributed to stones left behind in the common bile duct. In my experience these symptoms are almost limited to late cases in which delay before removal of the gall-bladder has already allowed infection to spread to the common bile duct. Cholangitis may then persist or recur, perhaps with exacerbations, even after drainage of the common bile duct, as well as after the removal of the gall-bladder. Medical treatment will generally cure these symptoms, and should be well tried before submitting the patient to another operation, which may not discover any stone or obstruction in the bile ducts. Recurrence of symptoms after operation may be due to residual infection in liver or pancreas, to stones overlooked in the ducts, to errors in diagnosis-such as overlooking osteo-arthritis of the spine-or to spastic or mucous colitis. 